\title{
ÉTOILES DOUBLES RÉVÉLÉES PAR LEURS MOUVEMENTS \\ PROPRES COMMUNS
}

\author{
A. N. DEUTSCH \\ Pulkovo Observatory, Leningrad U.S.S.R.
}

L'observatoire de Poulkovo a publié dans plusieurs mémoires les mouvements propres des 50000 étoiles jusqu'à la 15-me grandeur photographique obtenus par l'astrographe de la Carte du Ciel. Dans la présente étude nous avons utilisé 74 aires de Kapteyn $[1,2]$ et 25 autres aires ayant au centre les amas stellaires, les nébuleuses planétaires, les Novae etc $[3,4,5,6,7,8]$. Nous avons profité en outre de résultats du catalogue de Radcliffe [9] qui nous a fourni 41 aires de Kapteyn de plus. Donc on peut admettre que nous avons examiné 200 degrés carrés c'est à dire $\frac{1}{200}$ de la sphère céleste.

Les données du catalogue IDS [10] nous ont permis d'identifier 314 étoiles doubles ou multiples dont les mouvements propres étaient connus. Multipliant cette quantité par 200 nous recevons pour toute la sphère 63000 étoiles - ce qui est conforme avec le nombre total 64000 des étoiles du catalogue IDS. Cela veut dire que notre échantillon des objets d'observation est assez représentatif.

Dans la Tableau I qui suit nous donnons les nombres des étoiles doubles reparties par quatre classes - sûres, possibles, douteuses, non doubles - d'après la ressemblance

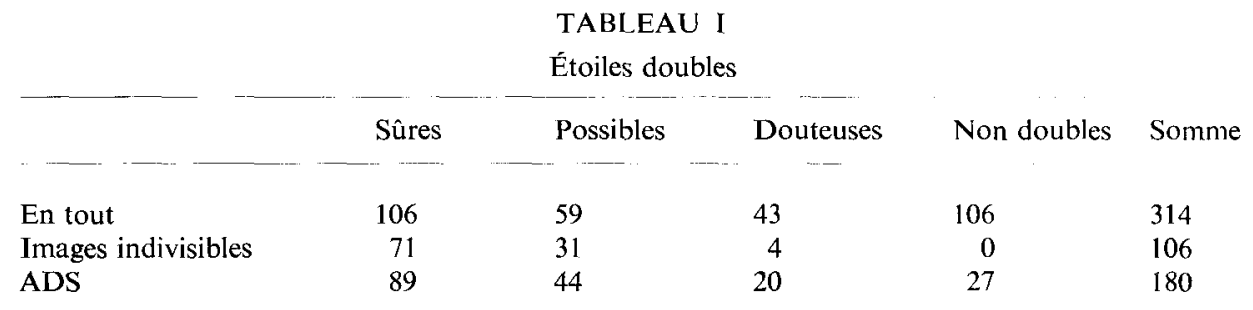

des mouvements propres des composants de la couple. En cas des triples ou multiples étoiles on a conté chaque compagnon à part.

L'échelle de l'astrographe $\left(60^{\prime \prime}=1 \mathrm{~mm}\right)$ ne permet pas de dédoubler les images des couples serrées $\left(\varrho=3^{\prime \prime}-6^{\prime \prime}\right)$, ce qui dépend, bien entendu, de la magnitude des étoiles. Nous donnons dans la seconde ligne de la tableau les nombres de pareilles couples. Elles appartiennent principalement aux deux premières classes. Si les mouvements propres sont très petits en proportion des erreurs probables $( \pm 0$ " $003- \pm 0.006)$ nous devrions en premier lieu nous contenter du critère de la distance $\varrho$.

Selon notre analyse la grande partie des étoiles 'non doubles' a été trouvée par les observateurs qui ont utilisé les clichés de la Carte du Ciel, comme Pourteau, Baillaud, Smart et Stein. Leurs mesures se rapportent aux étoiles doubles faibles de 11-13 mg et écartées de $10^{\prime \prime}-15^{\prime \prime}$. Dans la classe 'non doubles' se trouvent aussi beaucoup des 
compagnons des étoiles triples et multiples designées dans le catalogue ADS. La dernière ligne de la tableau montre les nombres des étoiles de ce catalogue, qui font plus de la moitié de la somme entière.

Quoique les résultats de cet examen ne sont ni trop exacts ni complets, nous sommes en droit de conclure qu'à peu près un tiers des étoiles contenues dans IDS ne peut être traité comme doubles, triples ou multiples systèmes.

\section{Bibliographie}

[1] Deutsch, A. N.: 1940, Publ. Obs. Poulkovo, 55.

[2] Deutsch. A. N.: 1961, Bull. Obs. Poulkovo 168, 79.

[3] Lavdovsky, V. V.: 1961, Publ. Obs. Poulkovo 73, 7.

[4] Orlova, O. N.: 1965, Bull. Obs. Poulkovo 176, 219.

[5] Orlova, O. N.: 1966, Bull. Obs. Poulkovo 179, 115.

[6] Kadla, Z. I.: 1966, Bull. Obs. Poulkovo 181, 93.

[7] Sokolova, V. A.: 1971, Publ. Obs. Poulkovo 78, sous presse.

[8] Bronnicova, N. M.: 1971, Publ. Obs. Poulkovo 78, sous presse.

[9] Knöx-Shaw, H. and Scott Barrett, H. S.: 1934, Radcliffe Catalogue, Oxford.

[10] Jeffers, H. and van den Bos, W.: 1963, Publ. Lick Obs. 21.

\section{Discussion}

Couteau: Au sujet des étoiles Pourteau dont vous avez parlé, j’ai constaté au hasard de mes observations que beaucoup d'entre elles n'existent pas. Il y en a 5000 dans l'IDS. La plupart sont de magnitude 13, bien des fois j'ai regardé le ciel et j'ai rarement vu des étoiles Pourteau. Je ne veux pas dire que réellement elles n'existent pas, je peux dire que je ne les ai pas vues là où elles auraient dû être. Je n'ai jamais posé la question à Pourteau, je ne sais pas comment il a fait son catalogue, je crois qu'il a dũ le constituer d'après la carte du ciel sans vérifier avec les instruments.

Deutsch: Dans la carte du ciel, il est arrivé quelque fois que deux mesures d'une même étoile aient été donneés comme une étoile double. La confusion des étoiles doubles pouvait-être plus grande dans les régions de la Voie Lactée, ce que nous avons remarqué.

Couteau: Pour Baillaux, c'est un peu la même chose, il a pris ses étoiles doubles dans la carte du ciel. Beaucoup existent, c'est entendu, mais elles n'ont jamais été vérifiées de visu ce qui est très dangereux.

Muller: A la suite de l'intervention de Monsieur Couteau, il convient de préciser que les faux couples carte du ciel ont été 'découverts' sur les positions publiées et non sur les clichés. Ce qui est confirmé par Monsieur R. Jonckheere puisque des positions imprimées avec une petite différence en raison d'une erreur matérielle, ont donné naissance à de faux couples.

Lacroute: Je voudrais faire une communication au sujet du catalogue astrographique. Actuellement nous projetons en France de dépouiller les zones françaises pour avoir des positions anciennes du catalogue photographique. C'est un travail que l'on compte faire en utilisant les positions de l'AGK2AGK3, mais comme il a été dit abondamment le catalogue photographique fourmille d'erreurs il est absolument impensable d'examiner toutes ces erreurs. La solution que nous comptons adopter est d'utiliser le recouvrement des clichés, de signaler comme 'sûres' les étoiles qui ont été obtenues au moins deux fois de façon concordante et pour les autres de les signaler comme 'une fois obtenues'. On saura que ce n'est pas sûr. Il est impossible de discuter tous les résultats; les mesures sûres pourront rendre service.

Jonckhecre: Je suis entièrement d'accord avec ce que dit Monsieur Couteau. Depuis 1917 j'ai attiré plusieurs fois l'attention, mais en vain, sur l'incertitude des étoiles doubles retirées des Catalogues de la carte du ciel, c'est pourquoi je les ai rejetées de mon premier Catalogue général publié en Angleterre.

Les coordonnées rectangulaires de ces catalogues peuvent donner n'importe quoi, même des couples de $0^{\prime \prime} 06$ de séparation. (J.O. 1954, p. 31). Il s'agit en fait de deux mesures différentes d'une même étoile. Même pour des séparations de $10^{\prime \prime}$ la réalité de ces 'Étoiles Doubles' n'est pas certaine. 
Quant à la reproduction des cartes, des coups de poinçon ont quelquefois été donnés à la main sur les plaques de cuivre pour accentuer l'impression d'étoiles sur le papier.

Pour certaines zones la moitié des étoiles doubles relevées dans ces catalogues n'existent pas dans le ciel. Elles se trouvent malheureusement dans l'Index Catalogue of Visual Double Stars.

Lacroute: Quant on a affaire à une documentation ancienne et peu sûre, c'est seulement la redondance qui permet d'affirmer quelque chose. 